\title{
Correction to: Endocrine Disease and Cognition
}

\section{Correction to:}

Chapter 8 in: D. Hedges et al., The Brain at Risk, https://doi.org/10.1007/978-3-030-14260-5_8

The original version of the book, chapter 8 was published with an incorrect abstract. The correction chapter has been updated with the changes.

The updated version of this chapter can be found at https://doi.org/10.1007/978-3-030-14260-5_8 